\title{
Phage therapy potentiates second-line antibiotic treatment against pneumonic plague
}

Yaron Vagima ${ }^{1}$, David Gur ${ }^{1}$, Moshe Aftalion ${ }^{1}$, Sarit Moses $^{1}$, Yinon Levy ${ }^{1}$, Arik Makovitzki², Tzvi Holtzman², Ziv Oren ${ }^{1}$, Yaniv Segula², Ella Fatelevich², Avital Tidharr, Ayelet Zauberman $^{1}$, Shahar Rotem ${ }^{1}$, Emanuelle Mamroud ${ }^{1 *}$ and Ida Steinberger-Levy ${ }^{1 *}$

${ }^{1}$ Department of Biochemistry and Molecular Genetics, Israel Institute for Biological Research, Ness-Ziona, Israel. ${ }^{2}$ Department of Biotechnology, Israel Institute for Biological Research, Ness Ziona, Israel.

Short title: Phage therapy against pneumonic plague

Conflict of interest: The authors declare that no conflicts of interest exist.

Correspondence: Ida Steinberger-Levy Ph.D. idasl@iibr.gov.il and Emanuelle Mamroud

Ph.D. emmym@iibr.gov.il. Department of Biochemistry and Molecular Genetics, Israel Institute for Biological Research, P.O. Box 19, Ness-Ziona, 74100, Israel. Tel: +972-8-

9381283, Fax: +972-8-9381544 


\section{Abstract}

2 Plague pandemics and outbreaks have killed millions of people during the history of

3 humankind. The disease, caused by Yersinia pestis bacteria, can currently be treated

4 efficiently with antibiotics. However, in the case of multidrug-resistant (MDR) bacteria,

5 alternative treatments are required. Bacteriophage (phage) therapy has shown efficient

6 antibacterial activity in various experimental animal models and in human patients

7 infected with different MDR pathogens. Herein, we evaluated the efficiency of $\phi A 1122$

8 and PST phage therapy, alone or in combination with second-line antibiotics, using a well-

9 established mouse model of pneumonic plague. Phage treatment significantly delayed

10 mortality and limited bacterial proliferation in the lungs. However, the treatment did not

11 prevent bacteremia, suggesting that phage efficiency may decrease in circulation. Indeed,

12 in vitro phage proliferation assays indicated that blood has inhibitory effects on lytic

13 activity, which may be the major cause of treatment inefficiency.

14 Combining phage therapy and second-line ceftriaxone treatment, which are individually

15 insufficient, provided protection that led to survival of all infected animals, presenting a

16 synergistic protective effect that represents a proof of concept for efficient combinatorial

17 therapy in an emergency event of a plague outbreak involving MDR Y. pestis strains. 


\section{Author summary}

22 Plague, caused by Yersinia pestis bacteria, can be efficiently treated with antibiotics.

23 However, alternative therapies for the case of natively evolved or maliciously generated

24 antibiotic-resistant $Y$. pestis must be developed. Due to the global increase in antibiotic

25 resistance, there is renewed interest in examining the effectiveness of bacteriophage-

26 based alternative therapies. Here, using a mouse model of pneumonic plague, we

27 demonstrate that phage treatment significantly delayed mortality. By monitoring

28 bioluminescence of engineered $Y$. pestis strain and live bacterial counts, we show that

29 phage therapy effectively inhibited bacterial proliferation in the lung but not in blood. In

30 vitro analyses showed decreased phage activity in the presence of blood, which probably

31 explains the low efficacy of phage treatment alone. Because combination therapies will

32 be used in an emergency situation, we tested the efficacy of $Y$. pestis-lysing phages as

33 adjunctive therapy with a second-line antibiotic, ceftriaxone.

34 Whereas each individual treatment was insufficient, the combination provided effective

35 protection and rescued all infected animals. These results clearly demonstrated the

36 synergistic effect of combined phage and antibiotic therapy and represent a proof of

37 concept for this alternative therapy against multidrug-resistant $Y$. pestis strains.

39 Key words: phage therapy, antibiotic therapy, ceftriaxone, Yersinia pestis, plague,

40 antibiotic resistance, $\phi A 1122$, PST, phage-antibiotic combination. 


\section{Introduction}

43 Yersinia pestis, a gram-negative bacterium and the etiological agent of plague, is a lethal

44 pathogen that has led to three world pandemics throughout human history [1,2].

45 Although natural plague outbreaks are rare, plague is considered a reemerging disease

$46[3,4]$. A recent large outbreak occurred in Madagascar in 2017, where many patients

47 presented with the pneumonic form of the disease $[5,6]$. Due to its lethality and the

48 potential for person-to-person infectivity, Y. pestis is classified by the Centers for Disease

49 Control and Prevention (CDC) as a tier-1 select agent [1,7]. Natural exposure of humans

50 to the bacteria may occur through a carrier flea bite, leading to bubonic or septic plague

51 that can be further developed into a secondary pneumonic plague, or by air transmission,

52 leading to primary pneumonic plague [1]. Pneumonic plague is a rapidly deteriorating

53 disease that, if not immediately treated with the recommended antibiotic, leads to death

$54 \quad[1,3]$.

$55 Y$. pestis strains are usually susceptible to the recommended antibiotics. However, several

56 strains isolated from rodents or from plague patients have demonstrated antibiotic

57 resistance against first-line antibiotics such as streptomycin [3,8-11]. Moreover, a major

58 concern exists regarding the possibility of the generation and usage of antibiotic-resistant

$59 Y$. pestis strains in a bioterror event $[3,4,7]$. Currently, no licensed plague vaccine is

60 available in Western countries [4,12], thus emphasizing the importance of developing

61 alternative treatments such as bacteriophage (phage) therapy. 
62 Lytic phages are bacterial viruses that demonstrate high specificity toward their hosts.

63 Phage replication inside the host bacteria leads to bacterial lysis and cell death, releasing

64 progeny virions in the so-called "lytic cycle". The potential of using phages for

65 antimicrobial therapy has been known for more than a century, since phages were

66 independently identified by Frederik Twort (in 1915) and Felix d'Herelle (in 1917) [13].

67 Their advantages of selective and efficient bacterial killing, self-replication in the presence

68 of the host, safety as treatments and simple and inexpensive preparation led to their

69 application in the 1920 s as a treatment against various infectious diseases, such as

70 dysentery and cholera $[13,14]$. However, the appearance of commercialized antibiotics in

71 the late 1940s changed the paradigm, and Western countries preferred the usage of

72 antibiotics, which are characterized chemical molecules, over the usage of phage, a

73 biological viral entity [13]. In contrast to the situation in the West, phage therapy

74 continued to serve as an acceptable remedy in the former USSR, Georgia and Eastern

75 European countries [15]. Recently, as antibiotic resistance has become a global threat to

76 public health, renewed interest in phage therapy has emerged in Western countries.

77 Phage efficiency and safety have been investigated using various bacterial-infected

78 animal models and by conducting human clinical trials [16]. Moreover, an increasing

79 number of case reports have described successful compassionate-use treatment of

80 multidrug-resistant (MDR) bacterial-infected patients by administration of antibiotics in

81 combination with lytic phages, selected in a personalized manner to lyse the isolated MDR

82 pathogen (see $[17,18]$ and recent reviews $[16,19])$. 
83 In the present study, we evaluated the potential of phage therapy against pneumonic

84 plague using a well-established mouse model. The therapeutic potential of treatment

85 with the $Y$. pestis-specific lytic phages $\phi A 1122$ and PST was assessed alone, as a dual

86 cocktail, or in combination with the second-line broad-spectrum cephalosporin antibiotic

87 ceftriaxone.

89 Results

\section{Intranasal administration of $\phi A 1122$ leads to high phage titers in}

91 the lungs

92 Pneumonic plague involves $Y$. pestis dissemination from the lung to internal organs and

93 blood [20]. Thus, for efficient phage treatment, phage distribution in those tissues is a

94 prerequisite. Therefore, we tested the tissue distribution of the $Y$. pestis-specific lytic

95 phage $\phi A 1122$ in C57BL/6J mice and examined its dependency on the administration

96 route. Mice were inoculated intranasally (IN) or intraperitoneally (IP) with a single dose

97 of phage suspension ( $1 \times 10^{9}$ plaque-forming units (PFU)/mouse). As shown in Fig 1, phages

98 reached all tested tissues (blood, lung, spleen and liver) within half an hour. The IN route

99 seemed advantageous for the treatment of pneumonic plague, as it led to the highest

100 phage concentration in the lung ( $1 \times 10^{9}$ PFU/lungs, Fig 1B). Moreover, high phage

101 concentrations were maintained in the lungs for at least 4 days (Fig 1B). Thus, we further

102 tested the potential of intranasal treatment with $\phi A 1122$ against $Y$. pestis airway 103 infection. 
104

A

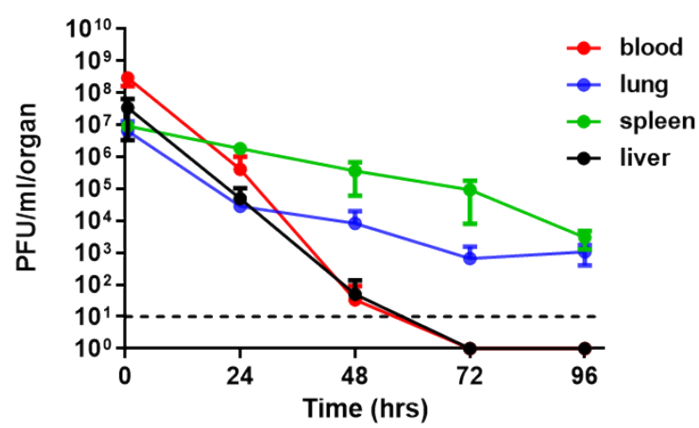

B

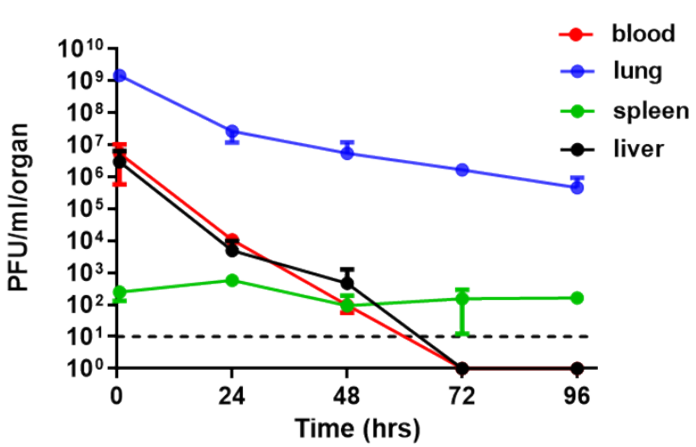

Fig 1. Pharmacokinetic analysis of $\phi A 1122$ in naïve mice. A single dose of $\phi A 1122$ phage suspension ( $\left.1 \times 10^{9} \mathrm{PFU}\right)$ was administered to naive $\mathrm{C} 57 \mathrm{BL} / 6 \mathrm{~J}$ mice, either by (A) IP injection (0.5 $\mathrm{ml}$ ) or (B) via the IN route $(35 \mu \mathrm{l})$. For each administration route, $\mathrm{n}=3$ for each time point. Phage titration was performed using a spot assay test. Each dot represents the mean value in terms of PFU/organ (lung - blue dots, spleen - green dots, liver - black dots) or PFU/ml blood (red dots). Bars represent the standard deviation (SD).

115 Iytic phage $\left(1 \times 10^{9} \mathrm{PFU} / \mathrm{mouse}\right)$ at $5 \mathrm{~h}$ post IN infection with a lethal dose of $10 \operatorname{LD}_{50} \mathrm{Y}$. 116 pestis strain Kim53.

117 As shown in Fig 2A, Y. pestis-infected control mice died within 3-4 days post infection (pi), 118 showing a mean time to death (MTTD) of 3 days. In contrast, phage-treated mice showed 119 a significant delay in time to death, which occurred within 4-7 days pi, with a MTTD of 5 120 days. 
121 To assess $Y$. pestis loads in the lungs of treated mice, we infected mice with a luminescent

$122 Y$. pestis derivative (Kim53-luxCDABE), harvested the lungs at days 2 and 4 pi and

123 visualized bacterial loads using an in vivo imaging system (IVIS). We found that at $48 \mathrm{~h} \mathrm{pi}$

124 (hpi), lungs from the nontreated control mice showed significantly higher levels of

125 luminescence that those from phage-treated mice (Fig 2, B-C). Bacterial live counts in the

126 lungs aligned with the IVIS results, demonstrating $>2$ orders of magnitude higher bacterial

127 loads in control vs. phage-treated mice at 48 hpi (Fig 2D). Similarly, inhibition of Y. pestis

128 growth in the lungs of phage-treated mice correlated with high concentrations of $\phi A 1122$

129 (Figs 2D and 2F, respectively).

130 Next, we assessed $Y$. pestis dissemination into the blood. During the first $48 \mathrm{~h}$, the

131 bacterial concentration in the blood of the control mice reached $\sim 10^{4}$ colony-forming

132 units (CFU)/ml, while no bacteria were detected in the blood of phage-treated mice (Fig

$1332 \mathrm{E})$. However, in the following days, bacteria also appeared in the circulation of phage-

134 treated mice (Fig 2E). The appearance of $Y$. pestis in the blood negatively correlated with

135 the phage amount, which was low at $48 \mathrm{hpi}$ and decreased further to under the limit of

136 detection at $96 \mathrm{hpi}$ (Fig 2F). We therefore concluded that while $Y$. pestis growth in the

137 lungs of treated mice was restricted, the reduction in phage amount in the blood during

138 disease progression enabled Y. pestis dissemination/propagation in the circulation, which

139 finally led to mouse death (Fig 2A). 
A

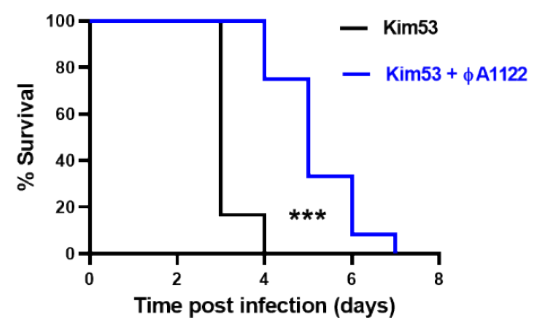

D

\section{efficacy}

B
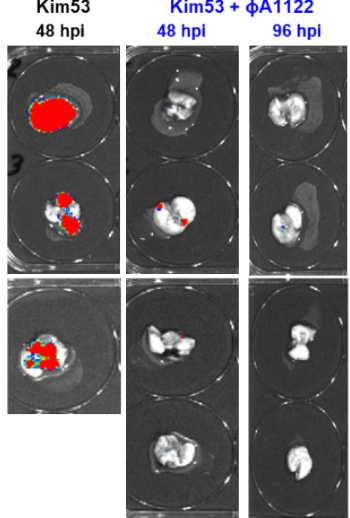

E

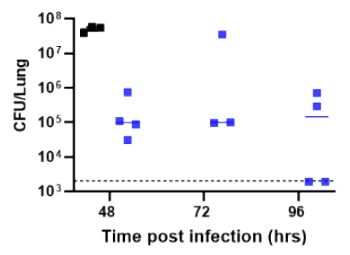

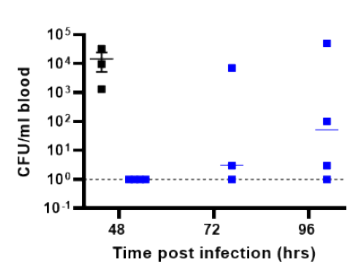

C

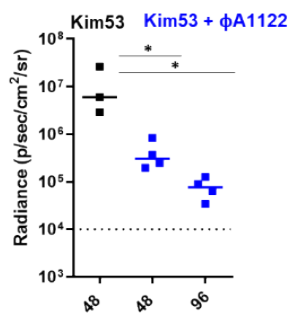

Time post infection (hrs)

.

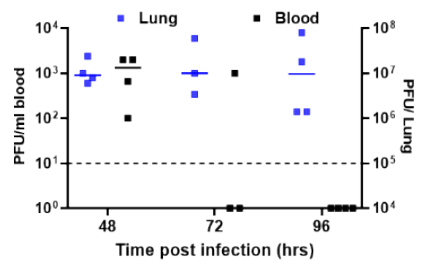

Fig 2. Intranasal administration of a single-dose phage treatment delayed disease progression. $\mathrm{C} 57 \mathrm{BL} / 6 \mathrm{~J}$ mice were intranasally infected with $10 \mathrm{xLD}_{50} Y$. pestis Kim53 strain (A) or Kim53-lux luminescent strain (B-F). For phage treatment, one dose of $1 \times 10^{9} \phi A 1122 \mathrm{PFU} /$ mouse was IN administered at $5 \mathrm{hpi}$. Control mice were subjected to intranasal administration of PBS. A. Survival curves of control mice (black line, $n=6$ ) and phage-treated mice (blue line, $n=12$ ). B-C. Control $(n=3)$ and phage-treated mice $(n=4)$ were anesthetized at the indicated time points post $Y$. pestis infection. Lungs were harvested, and imaging was performed using IVIS as detailed in the Materials and Methods section. D-E. The bacterial load in the lungs and blood was quantified by plating serial dilutions of tissue homogenate/blood on BHIA plates supplemented with $200 \mu \mathrm{g} / \mathrm{ml}$ ampicillin and counting the colonies. F. Phage titration was performed by the spot assay technique as detailed in the Materials and Methods section. Each point represents the phage load in the lungs (blue squares, total PFU/lung) or blood (black squares, PFU/ml) of an individual mouse. Horizontal bars represent median values. Dotted lines mark the limit of detection. Statistically significant differences between groups are denoted by asterisks $\left(*, P<0.05\right.$; ${ }^{* * *} P<0.0001$, [log rank [Mantel-Cox] test]). Bars indicate standard errors of the means.

\section{Multiple-dose phage administrations did not improve treatment}


162 To improve phage treatment and increase phage load in the blood and internal organs,

163 we designed additional treatment regimens (Fig 3A). Pharmacokinetic analysis showed

164 that in comparison to IN administration, IP injection of $\phi A 1122$ led to higher phage

165 concentrations in the blood, spleen and liver (Fig 1); thus, we added IP injection doses

166 after the initial IN treatment. Moreover, since phages are rapidly cleared from the blood

167 and liver (Fig 1), we provided serial phage treatment in 24-h intervals for 6 days pi. As

168 depicted in Fig 3B, phage treatment was effective in delaying mouse mortality in

169 comparison to that in the nontreated group, as shown above (MTTD $=6$ and 3 days,

170 respectively). However, no differences were found among the various phage-treated

171 groups. Thus, increasing phage loads in internal organs during the course of the disease

172 did not improve infection outcomes, suggesting that $\phi A 1122$ lytic activity in the blood

173 might be insufficient. 


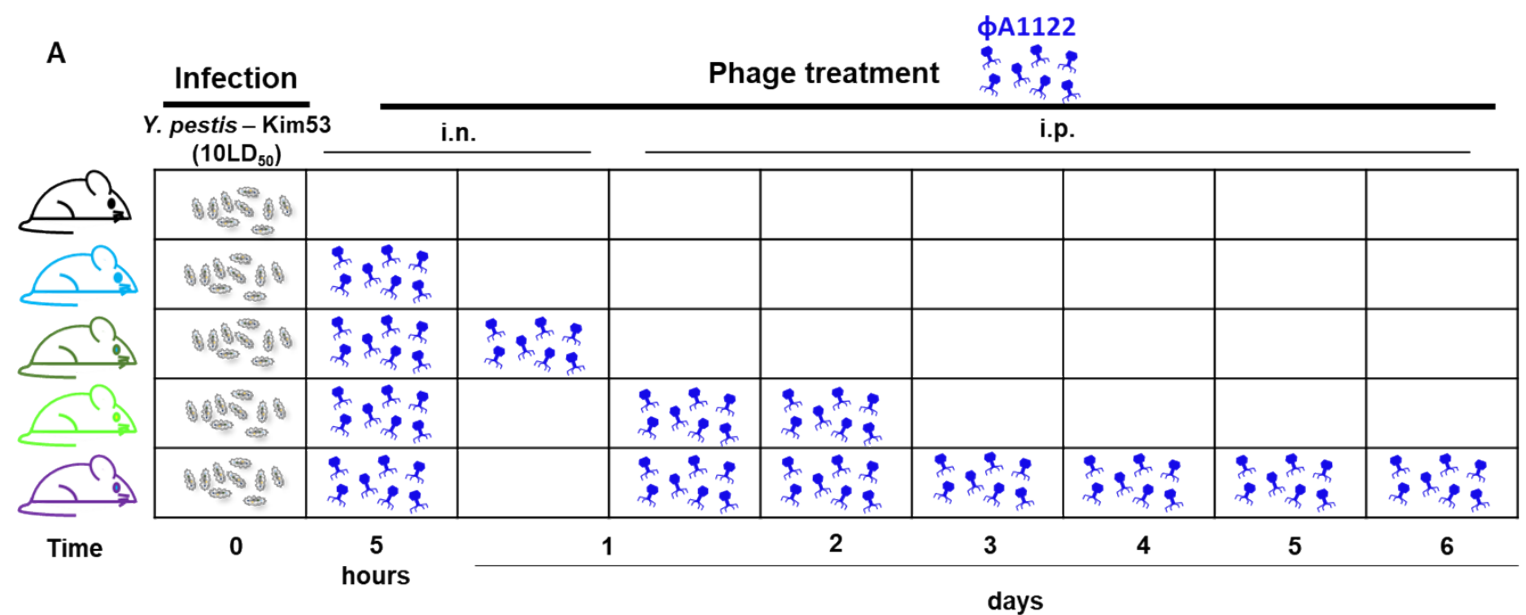

B

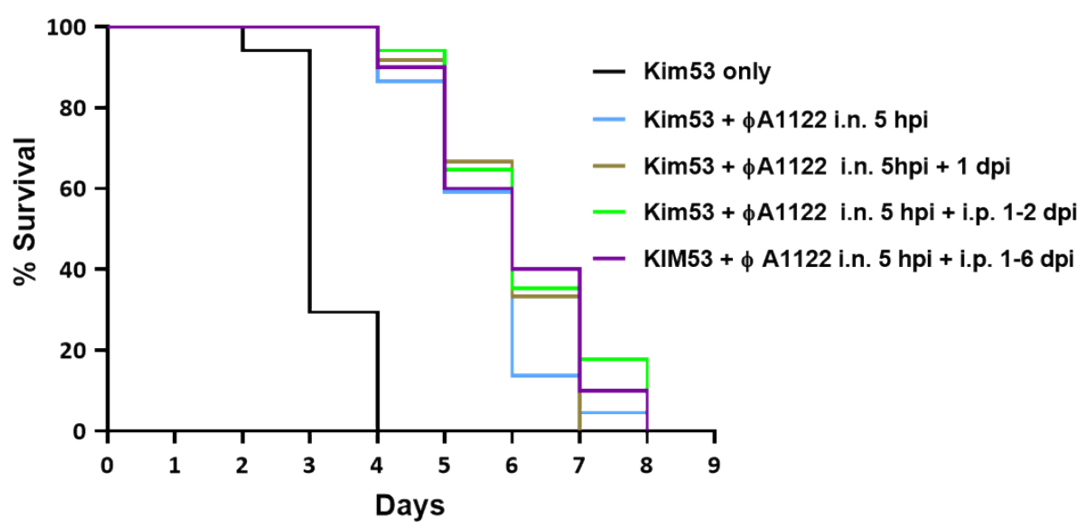

Fig 3. Multiple-dose $\phi A 1122$ phage treatments did not improve treatment efficacy. Schematic representation of mouse treatment regimens $(A)$ and survival curves (B). C57BL/6J mice were IN infected with $10 \times L_{50} Y$. pestis Kim53. For IN phage treatment, mice were inoculated with $35 \mu \mathrm{l}$ of $1 \times 10^{9}$ \$A1122 PFU; IP injection included administration of $0.5 \mathrm{ml}$ of $1 \times 10^{9}$ \$A1122 PFU. The treatment regimens were as follows: 1 dose of IN $\phi A 1122$ at $5 \mathrm{hpi}(\mathrm{n}=22$; light blue line), 2 doses of IN $\phi A 1122$ at $5 \mathrm{hpi}$ and $24 \mathrm{hpi}$ ( $\mathrm{n}=12$, olive green line), 1 dose of IN $\phi A 1122$ at $5 \mathrm{hpi}+$ IP injections at $24+48 \mathrm{hpi}(\mathrm{n}=17$, green line) and 1 dose of IN $\phi A 1122$ at $5 \mathrm{hpi}+\mathrm{IP}$ injections for 6 days, every $24 h$ ( $n=10$, purple line). Control mice: $n=17$, black line. B. Statistically significant differences between the control group and phage-treated groups are denoted by asterisks 
188 Since the addition of IP administered phage doses did not improve mouse survival (Fig
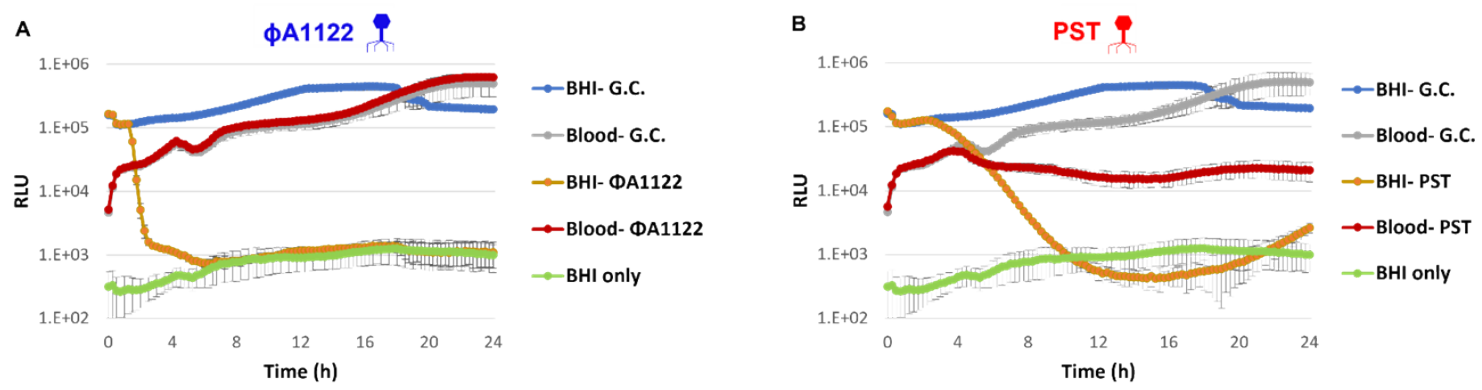

Fig 4. PST is more potent than $\phi A 1122$ in the presence of blood. Phage-based lysis assays were performed with the bioluminescent $Y$. pestis strain EV76-lux $\left(10^{7} \mathrm{CFU} / \mathrm{ml}\right)$ suspended in $\mathrm{BHI}$ broth (blue and orange lines) or in mouse whole blood (gray and red lines). The $Y$. pestis strain was infected with $\phi A 1122$ or PST phages $\left(10^{6} \mathrm{PFU} / \mathrm{ml}\right.$; multiplicity of infection [MOI] $\left.=0.01\right)$. Bioluminescence (RLU) was measured at $37^{\circ} \mathrm{C}$ in 15 min intervals for $24 \mathrm{~h}$ using a Spark $10 \mathrm{M}$ plate reader. The experiment was performed in biological duplicates (using blood pooled from 3 mice for each experiment), and the results are representative of one experiment. Values are the 
211 the blood (to under the LOD by $72 \mathrm{~h}$ post administration, Fig 1), PST presence in the blood

212 was detected even at 4 days post IN or IP administration.
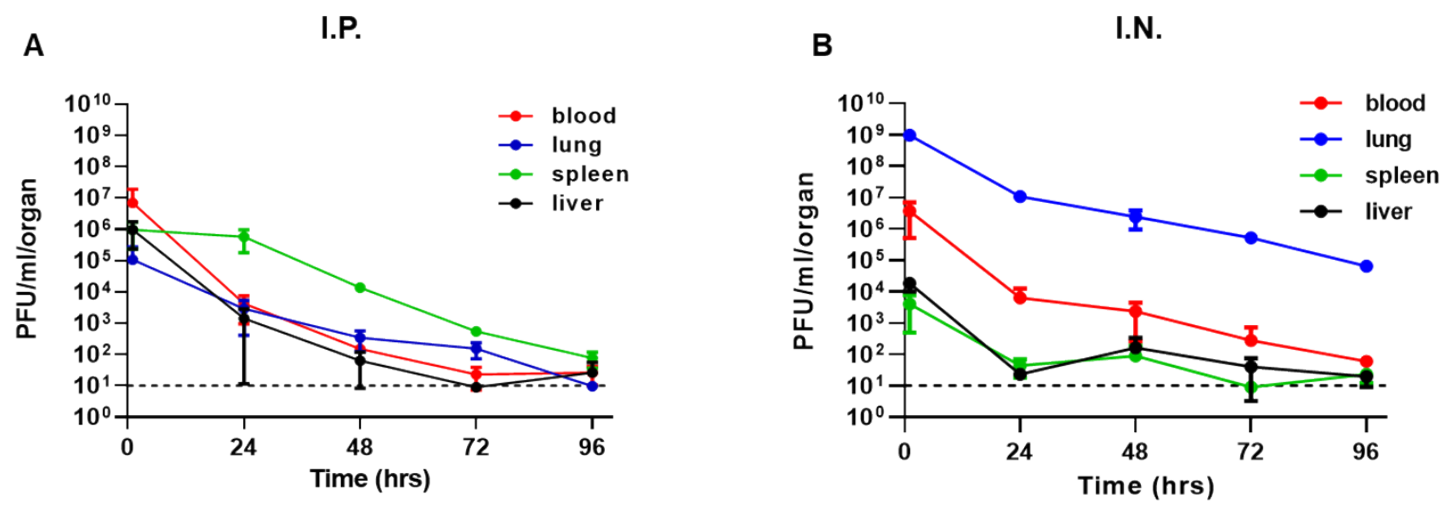

214 Fig 5. Pharmacokinetic analysis of PST in naïve mice. The experiment was performed as

215 described in the Fig 1 legend.

\section{Comparing the protective potential of PST and $\mathbf{\phi A 1 1 2 2}$}

218 Next, we compared the therapeutic potential of the phages. The treatment regimen with

219 PST and $\phi A 1122$ is schematically outlined in Fig 6A, and the results indicated that both

220 phages are comparable in their antiplague protective abilities (Fig 6B). This analysis

221 indicates that phage therapy alone is limited in its ability to protect against acute

222 pneumonic plague. 


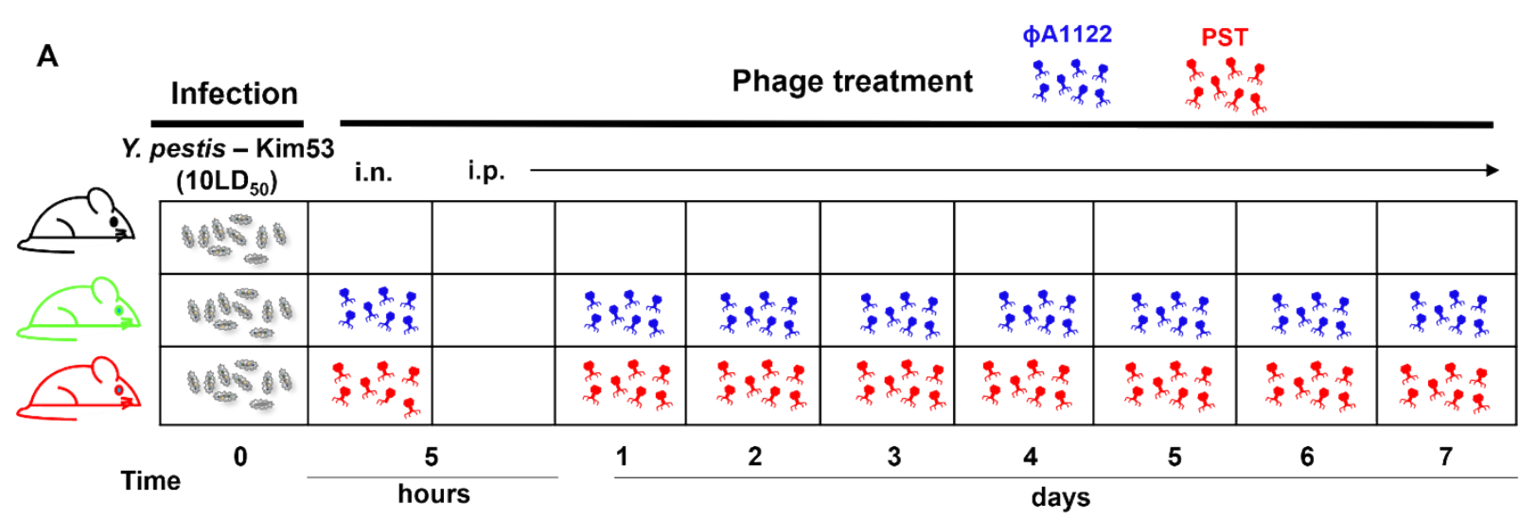

B

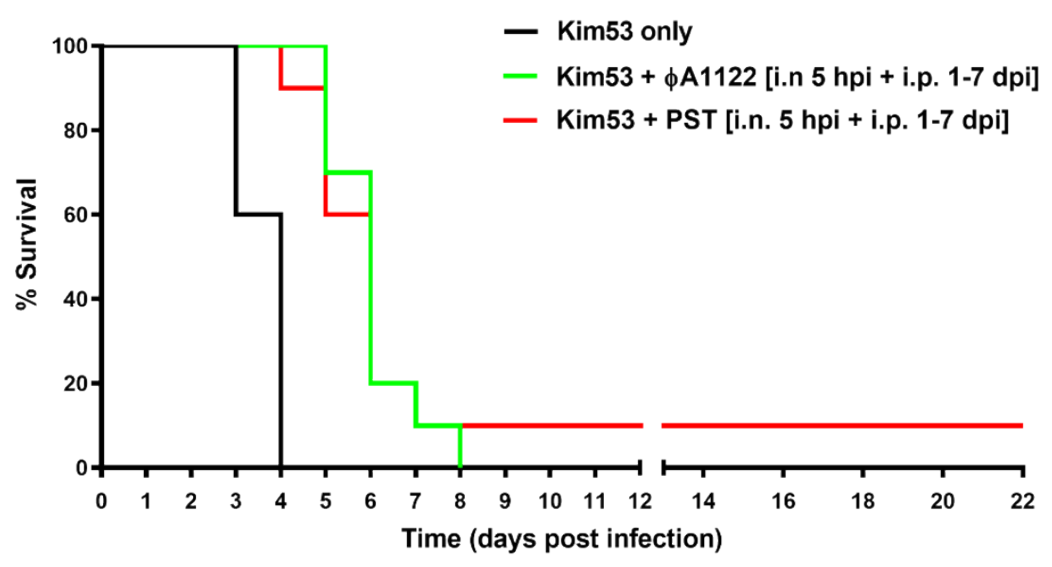

Fig 6. Treatment with $\$ A 1122$ or PST against pneumonic plague has similar outcomes. $10 x \mathrm{LD}_{50}$ Y. pestis Kim53 (gray symbols), followed by PBS (control group) or phage administration. Each dose of PST (red phage symbol) or $\phi A 1122$ (blue phage symbol) suspension contained $1 \times 10^{9}$ phages. B. Survival curve. The mouse groups were as follows: no phage ( $n=5$, black line), IN phage administration at $5 \mathrm{hpi}$ followed by IP injections every $24 \mathrm{~h}$ at days 1-7 post bacterial infection

\section{Phage-ceftriaxone combination therapy is highly effective}

\section{3 against pneumonic plague}

234 In the event of a plague outbreak involving MDR bacteria, second-line antibiotics may be 
236 active against $Y$. pestis strains in vitro [1], but they are limited in their activity in vivo

237 [21,22]. To assess the therapeutic value of adjunctive phage therapy to second-line 238 antibiotic treatment, C57BL/6 mice were IN challenged with a high dose of $Y$. pestis Kim53

$239\left(100 \times L_{50}\right)$ and treated with phage, ceftriaxone or a combination of both (Fig $\left.7 \mathrm{~A}\right)$. For

240 phage treatment, we used a cocktail composed of both $\phi A 1122$ and PST ( $1 \times 10^{9}$ PFU of

241 each per dose). Antibiotic treatment with ceftriaxone rescued only $20 \%$ of the mice (Fig

242 7B), and as was found for treatment with PST or $\phi A 1122$ alone (Fig 6), treatment with the

243 phage cocktail delayed mortality; however, all mice succumbed to the infection (Fig 7B).

244 In contrast, the phage-antibiotic combination treatment was highly effective, leading to

245 the survival of all infected animals (Fig 7B) and clearance of the pathogen from the spleens

246 of all surviving animals at day 21 pi. In addition, surviving animals developed high serum

$247 \lg$ antibody titers against the protective $\mathrm{F} 1$ antigen (GMT=25,000 at 21 days pi), implying

248 that the combined treatment also enabled the development of adaptive immunity toward

249 Y. pestis. 


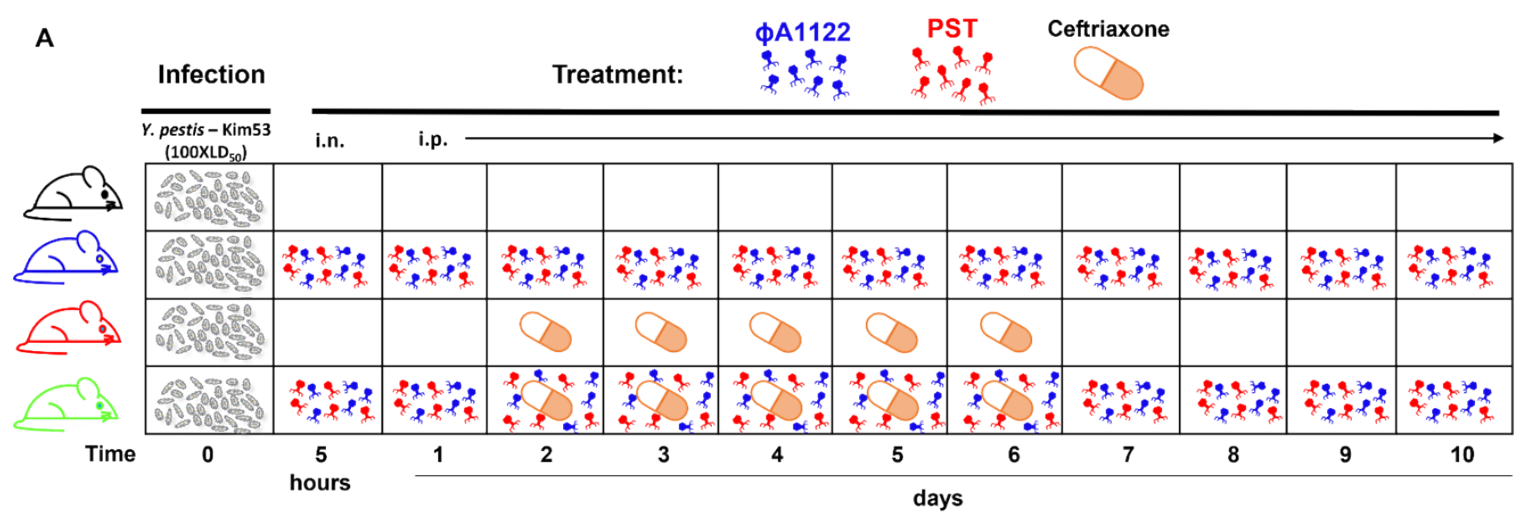

B

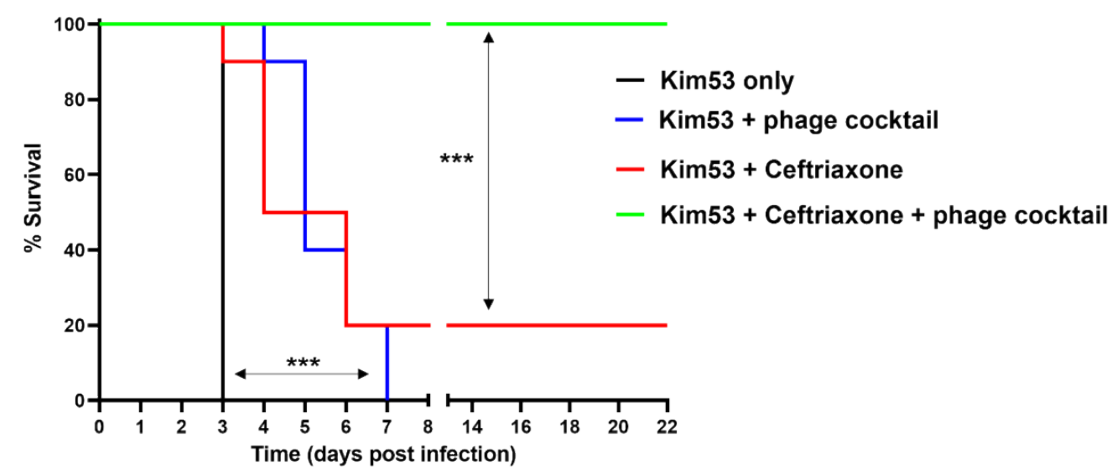

252 Fig 7. Effective rescue of infected mice by phage-antibiotic combination treatment.

253 C57BL/6J female mice were IN infected with $100 \times \mathrm{LD}_{50} \mathrm{Y}$. pestis Kim53. Mouse groups included 254 control nontreated $(n=4)$, phage-treated $(n=10)$, ceftriaxone-treated $(n=10)$ and phageceftriaxone combination-treated mice $(n=9)$. Phage treatment was performed using a phage cocktail composed of $\phi A 1122$ and PST $\left(1 \times 10^{9}\right.$ each phage/1 dose; $35 \mu$ for intranasal administration or $0.5 \mathrm{ml}$ for IP injection). Treatment included IN administration at $5 \mathrm{hpi}$ followed by IP injections at days 1-10, with 24-h intervals. Ceftriaxone was subcutaneously injected every $12 \mathrm{~h}$ on days 2-6 post bacterial infection. Mice were monitored for 22 days. Statistically significant differences are denoted by asterisks (***, $\mathrm{P}<0.001$; log rank [Mantek-Cox] test).

\section{Discussion}

263 The experience of the current emerging COVID-19 pandemic with its broad effects on

264 humankind has highlighted the need for preparedness for bacterial-derived outbreaks. In

265 this regard, a major concern is the appearance of highly transmissible MDR pathogens for

which current antibiotics may become ineffective. In the case of the plague-causing $Y$. 
267 pestis bacterium, antibiotics can effectively treat plague and prevent $Y$. pestis spread in

268 the population. However, a noncontrolled outbreak, which may cause high lethality, may

269 evolve in the case of the emergence and spread of natively derived or deliberately

270 engineered MDR strains. In such circumstances, clinicians will have to use less effective

271 second-line antibiotics for treatment. Adjunctive phage therapy may improve the

272 efficiency of such second-line antibiotics and serve as a safe alternative treatment in the

273 event of MDR infections.

274 The potential of phages as antiplague therapy was reported almost a century ago (in 1925)

275 by Felix D'Herelle, who injected $Y$. pestis-specific phage preparations into the buboes of

276 four plague patients, all of whom recovered [23]. However, following inconclusive results

277 obtained with additional phage treatment experiments and with the availability of

278 effective antibiotics, interest in developing phage-based therapy against plague

279 decreased. In one of the few studies in which the effectiveness of phage therapy against

280 plague was examined, Filippov and his colleagues assessed the outcome of phage therapy

281 with the $\phi A 1122$ phage in a mouse model of bubonic plague. The study demonstrated

282 that phage treatment was able to delay mouse mortality and provide protection to some

283 of the infected animals [24].

284 In the present study, we explored the potential of phage therapy ( $\$$ A1122 and PST) alone

285 and in combination with the second-line antibiotic ceftriaxone against pneumonic plague

286 using a mouse model. The $Y$. pestis-specific lytic phages $\phi A 1122$ and PST were chosen for

287 treatment as they are well characterized, and their genomic sequence is known, showing

288 no genes encoding integrase, known antibiotic resistance proteins or toxins $[25,26]$. The 
289 \$A1122 phage is well suited for use for treatment, as it was shown to be universal against

290 thousands of $Y$. pestis strains and thus is used by the CDC and the U.S. Army Medical

291 Research Institute of Infectious Diseases for $Y$. pestis diagnostics [26,27]. In addition,

292 \$A1122 and PST bind to different receptors, making their combination as a cocktail a

293 preferable formula to prevent the selection of phage-resistant mutants $[25,26]$.

294 To evaluate phage therapeutic potential, we infected C57BL/6J mice by intranasal

295 administration of a lethal dose of the $Y$. pestis Kim53 strain followed by treatment with

296 the \$A1122 and PST phages alone or as a cocktail. IN treatment with only one dose of

297 phage at 5 hpi led to a significant delay in mortality, which correlated with a lower

298 bacterial load in the lungs and slower kinetics of bacterial dissemination/propagation in

299 the blood that those in control nontreated counterparts (Fig 2). However, although

300 disease progression was delayed, phage administration was unable to provide protection,

301 and all treated animals succumbed to the infection (Figs 2-3). Delayed mortality seemed

302 to be associated with low phage concentrations in the circulation (Figs 1 and 5). However,

303 IP administration of additional phage doses to elevate phage titers in the blood did not

304 improve mouse survival in comparison to that with treatment with a single IN dose (Figs

3053 and 6). Therefore, the limited treatment efficiency may indicate a reduction in phage

306 Iytic activity in the presence of mouse blood (Fig 4), as we have recently observed in

307 human blood [28] and has been reported for other phages [29,30]. However, the PST

308 phage, which retained in vitro activity in the presence of blood, still yielded a similarly low

309 therapeutic efficiency in vivo as $\phi A 1122$ (Fig 6), indicating that additional limiting factors

310 exist. 
311 It is possible that the presence of $\phi A 1122$ and PST in the spleen and the liver, as reflected

312 by the pharmacokinetic analysis, may indicate phage clearance by the reticuloendothelial

313 system, whereby phages are not free to infect bacteria [31]. Similar observations for

314 \$A1122-treated bubonic plague mice led Filippov and his colleagues to suggest that the

315 phage and pathogen reside in different compartments in the spleen and liver; therefore,

316 the phage cannot infect and clear the bacterium [24,32]. Finally, the development of

317 phage resistance seems improbable in our model since it has been shown that mutations

318 in the $Y$. pestis lipopolysaccharide (LPS) phage receptors attenuate $Y$. pestis virulence [25]

319 and both $\phi A 1122$ and PST use the LPS receptor to infect the pathogen $[25,26]$.

320 Although phage treatment failed to save the infected animals, the significantly delayed

321 mortality encouraged us to evaluate the potential of phage as adjunctive therapy to

322 second-line antibiotics as an alternative countermeasure against MDR $Y$. pestis. It has

323 been shown in various case reports and animal model studies that combining antibiotics

324 with phages may enhance bacterial clearance, increase antibiotic penetration into

325 biofilms and decrease the emergence of phage resistance (see reviews [33,34]).

326 For the combined phage-antibiotic treatment, we used a phage cocktail composed of

327 \$A1122 and PST, gaining the high observed efficiency of $\phi A 1122$ of $Y$. pestis elimination

328 in the lungs and PST lytic activity in the presence of blood. Additionally, $\$$ A1122 and PST

329 bind to different sites in the LPS molecule [31,35] and thus targeting different receptors.

330 It is recommended to use phage cocktails composed of several lytic phages targeting

331 various receptors for efficient phage therapy, as this approach broadens the host range

332 and decreases the probability of phage resistance evolution. 
333 For the combined treatment, we used ceftriaxone, an antibiotic that has been shown to

334 have potent in vitro activity against $Y$. pestis strains [36] but limited ability to provide

335 protection in a mouse model of pneumonic plague [21,22]. Indeed, when mice were

336 treated with ceftriaxone as a single treatment, $80 \%$ of the animals succumbed to the

337 infection (Fig 7B). In contrast, the combined treatment of ceftriaxone and the phage

338 cocktail was highly effective, leading to the survival of all infected animals (Fig 7B) and

339 clearance of the pathogen from internal organs. Surviving animals developed high

340 antibody titers against the protective F1 capsular antigen, indicating the development of

341 adaptive immunity that probably contributed to the combined treatment efficacy.

342 In conclusion, our results suggest a synergistic effect between phage and antibiotic

343 treatment and highlight the potential of these combinations for emergency use even in

344 cases of acute infections such as pneumonic plague. Notably, training phages to efficiently

345 lyse bacteria in the presence of blood components may improve the therapeutic

346 outcome.

347 Further research on phage combinations with antibiotics as well as the potential for

348 resensitization to antibiotics may broaden the potential of phage therapy as an

349 alternative therapy against MDR pathogens.

351 Materials and methods

352 Bacteria, phages and growth media

\section{Table 1. Y. pestis strains used in the present work}




\begin{tabular}{|l|l|c|c|}
\hline Strain & Use & Virulence & Reference \\
\hline Kimberley53 & Challenge in animal & + & {$[37]$} \\
\hline Kimberley53-luxCDABE & IVIS imaging of & + & {$[20]$} \\
(Kim53-lux) & bacteria in the lungs & & {$[37]$} \\
\hline Kim53 $\triangle \mathrm{pPCD1} \triangle \mathrm{pPCP1}$ & Phage preparation & - & \\
(Kim53 $\triangle 70 \triangle 10)$ & and titration & & {$[28]$} \\
\hline EV76-IuxCDABE & Phage lysis assay & - & \\
\hline (EV76-lux $)$ & & & \\
\hline
\end{tabular}

355 The Kim53 and Kim53 $\Delta 70 \Delta 10$ strains were grown on Brain-Heart-Infusion Agar (BHIA;

356 241830, BD, USA), and Kim53-lux and EV76-lux were routinely grown on BHIA

357 supplemented with ampicillin $(200 \mu \mathrm{g} / \mathrm{ml}$, A0166, Sigma-Aldrich, Israel).

358 The $Y$. pestis-specific lytic phages used in this study were $\phi A 1122$ (accession no.

359 NC004777, kindly provided by professor Mikael Skurnik [38]), and PST (ATCC, cat. no.:

360 23207-B1; accession no. KF208315).

\section{Animal studies}

363 The study was performed in accordance with the Recommendations for the Care and Use 364 of Laboratory Animals (National Institutes of Health [NIH]). Animal experiments were 
365 performed in accordance with Israeli law and were approved by the Institutional Ethics

366 Committee for animal experiments (protocols no.: M-68-17, M-59-18, M-47-19, M-01-20,

367 M-72-20 and M-25-21). Experiments were performed in an animal biosafety level 3 (ABSL-

368 3) laboratory. Female 8- to 12-week-old mice (C57BL/6J; Invigo, Israel) were used for all

369 animal experiments.

370 For IN challenge, bacterial colonies of Kim53 or Kim53-luxCDABE were grown on heart

371 infusion broth (HIB, 238400, BD, USA) supplemented with $0.2 \%$ xylose and $2.5 \mathrm{mM} \mathrm{CaCl}_{2}$

372 (Sigma-Aldrich, Israel) at $28^{\circ} \mathrm{C}$ for $22 \mathrm{~h}$, as previously described [20]. Counting of CFU was

373 performed by plating $0.1 \mathrm{ml}$ of the appropriate culture dilutions on BHIA plates and

374 incubating them for $48 \mathrm{~h}$ at $28^{\circ} \mathrm{C}$. Prior to infection, mice were anesthetized with a mixture

375 of $0.5 \%$ ketamine $\mathrm{HCl}$ and $0.1 \%$ xylazine and then infected IN with $35 \mu \mathrm{l}$ of the bacterial

376 suspension containing $10 \times \operatorname{LD}_{50}$ or $100 \times \operatorname{LD}_{50} \operatorname{Kim} 53\left(1 \times I^{2} L_{50}=1,100\right.$ CFU [39]) per mouse.

377 For monitoring of bacterial dissemination to the lungs and blood, mice were anesthetized.

378 Blood was collected by cardiac puncture, and lungs were harvested and plated on BHIA

379 supplemented with streptomycin (100 $\mu \mathrm{g} / \mathrm{ml}$, S6501, Sigma-Aldrich, Israel).

380 For phage pharmacokinetic analysis, one dose of $\phi A 1122$ or PST phage solution ( $1 \times 10^{9}$

$381 \mathrm{PFU} /$ mouse) was IN administered to C57BL/6J mice (35 $\mu \mathrm{l})$ or delivered by IP injection (0.5

$382 \mathrm{ml}$ ). Mice were anesthetized prior to IN administration. For phage enumeration in organs,

383 mice ( $\mathrm{n}=3$ per time point and per administration route) were sacrificed, and blood was

384 collected by cardiac puncture followed by serial 10 -fold dilution in SM buffer $(0.1 \mathrm{M} \mathrm{NaCl}$,

$3858 \mathrm{mM} \mathrm{MgSO}_{4}, 50 \mathrm{mM}$ Tris- $\mathrm{HCl} \mathrm{pH} 7.5$ and $0.01 \%$ gelatin solution). The lungs, spleen and 
386 liver were harvested, washed in PBS and transferred to 1 well in 6-well microplates. The

387 spleen, liver and lungs were crushed in PBS (1 $\mathrm{ml}$ for spleen and liver and $2 \mathrm{ml}$ for lungs).

388 The lung extract was filtered through a $70 \mu \mathrm{m}$ cell strainer. The crushed tissues were

389 serially diluted 10 -fold in SM buffer. Phage titration of the diluted samples was performed

390 by the spot assay technique by dropping 3 drops of $10 \mu$ l samples on Kim $53 \Delta 70 \Delta 10$ lawns

391 grown on BHIA top agar containing $200 \mu \mathrm{g} / \mathrm{ml}$ streptomycin to prevent bacterial

392 contamination [30].

393 For bioluminescence imaging analysis of luciferase-expressing $Y$. pestis, mice were IN 394 exposed to $10 x \mathrm{LD}_{50}$ Kim53-lux followed by IN administration of $\phi A 1122$ or PBS (control group).

395 Photon emission from the lungs was visualized using an IVIS (Caliper Life Sciences, Hopkinton, 396 MA). Image acquisition was performed using the following settings: binning of 2 and acquisition 397 times of 1-4 min. The luminescence signals for all images were normalized and reported as 398 photons/second $/ \mathrm{cm}^{2} / \mathrm{sr}$ using Living Image ${ }^{\circledR} 4$ software.

399 For titration of anti-F1 IgG in serum, blood was collected from mice via the tail vein at day $21 \mathrm{pi}$, 400 and titers of IgG against F1 were determined by ELISA as previously described [40].

402 Phage treatment

403 Phages were applied as a single suspension (35 $\mu$ containing $10^{9}$ PFU of $\phi A 1122$ or PST) 404 or as a cocktail ( $\phi \mathrm{A} 1122+\mathrm{PST}, 10^{9} \mathrm{PFU}$ each). IN administration was conducted $5 \mathrm{~h}$ post $405 Y$. pestis infection. Phage IP injections ( $\left.0.5 \mathrm{ml}, 1 \times 10^{9} \mathrm{PFU} / \mathrm{mouse}\right)$ were conducted at $24 \mathrm{~h}$ 406 intervals (regimens described in the relevant figure legend). Prior to phage IN 
407 administration, mice were anesthetized by subcutaneous injection of $0.5 \%$ ketamine $\mathrm{HCl}$

408 and $0.1 \%$ xylazine mixed solution. Control mice were administered PBS.

\section{Antibiotic treatment}

411 Ceftriaxone (PHR-1382, Sigma-Aldrich, Israel) was injected subcutaneously twice per day

412 for 5 consecutive days, starting at $48 \mathrm{~h}$ post $Y$. pestis infection.

\section{Statistical analysis}

415 The results were analyzed by GraphPad Prism 8.2.0. Pairwise comparisons of mouse

416 groups were determined using a log rank (Mantel-Cox) test. A P value of 0.05 was

417 characterized as the significance threshold.

\section{Bioluminescence-based lysis assay}

420 Blood from 3 naive $\mathrm{C} 57 \mathrm{BL} / 6 \mathrm{~J}$ mice was pooled in citrate-containing tubes

421 (Vacutainer sodium citrate tubes, BD, USA). The Y. pestis EV76-lux strain was grown on

$422 \mathrm{BHIA}$ at $37^{\circ} \mathrm{C}$ for $48 \mathrm{~h}$. Bacterial colonies were suspended in PBS and inoculated (1:10;

423 vol:vol) in $\mathrm{BHI}$ broth or in mouse whole blood and transferred ( $90 \mu \mathrm{l} /$ well) into a 96 -well

424 transparent-bottom white microplate (Thermo Scientific Nunc: cat. no. 165306). Infection

425 was performed by adding $10 \mu \mathrm{l}$ of $\phi \mathrm{A} 1122$ or PST phage solution $(\mathrm{MOI}=0.01)$ or $10 \mu \mathrm{l}$ of 
426 SM buffer to the growth control wells. The bacterial growth curves were assessed by

427 tracking the bioluminescent signal (relative light units, RLU) of each well at $15 \mathrm{~min}$

428 intervals over $24 \mathrm{~h}$ using a SPARK $10 \mathrm{M}$ plate reader (Tecan). The temperature in all

429 experiments was $37^{\circ} \mathrm{C}$.

431 Phage preparation and purification

432 Purified bacteriophage stocks ( $\$ \mathrm{~A} 1122$ or PST phages) were prepared from phage-

433 infected cultures of nonvirulent $Y$. pestis Kim53 $\Delta 70 \Delta 10$. Phage lysates were prepared by

434 growing bacterial cultures in two 2 I Erlenmeyer flasks containing $500 \mathrm{ml}$ of $\mathrm{BH}$ inoculated

435 with overnight starter culture to obtain an initial $\mathrm{OD}_{600}$ of 0.05 . Bacterial cultures were

436 incubated at $200 \mathrm{rpm}$ at $28^{\circ} \mathrm{C}$ until $\mathrm{OD}_{660}=1$. Phage solution was added to the bacterial

437 culture $(\mathrm{MOI}=0.01)$, and the culture was further incubated under the same conditions for

438 another $4 \mathrm{~h}$ for $\phi A 1122$ or $24 \mathrm{~h}$ for PST. Culture lysates were centrifuged at 6,000 rpm for

$43910 \mathrm{~min}$ at $4^{\circ} \mathrm{C}$ followed by $0.45 \mu \mathrm{m}$ filtration for the removal of residual bacteria or

440 bacterial debris. Bacterial DNA was removed by endonuclease digestion using DENARASE

441 endonuclease (c-LEcta, Germany). Digestion was performed with $20 \mathrm{U} / \mathrm{ml}$ endonuclease

442 in the presence of $2 \mathrm{mM} \mathrm{MgCl}_{2}$ (Spectrum, New Brunswick, NJ) for $24 \mathrm{~h}$ at $4^{\circ} \mathrm{C}$ under

443 constant mild agitation. Sucrose (St. Louis, MO) was added to the phage solution to a final

444 concentration of $4 \%$. Ultrafiltration was performed using a 300 or 750 kDa (for $\phi A 1122$ or

445 PST purification, respectively) nominal molecular weight cutoff (NMWC) with a $1 \mathrm{~mm}$

446 diameter polyethersulfone (PES) hollow fiber membrane cartridge (Cytiva, Marlborough, 
447 MA). Using an ultrafiltration system, the phage solution was concentrated 10-fold by

448 volume and diafiltrated X5 against PBS (Biological Industries, Israel) supplemented with

$4494 \%$ sucrose. The ultrafiltrated phage solution was filtered again through a $0.45 \mu \mathrm{m}$ filter.

450 Endotoxin removal was performed twice by using a Toxin Eraser endotoxin removal kit

451 (GenScript, Piscataway, NJ) according to the manufacturer's guidelines. Elution was

452 performed with a PBS- $0.4 \%$ sucrose solution buffer followed by $0.2 \mu \mathrm{m}$ filtration. Residual

453 endotoxins in the purified phage preparations were determined by the Limulus

454 Amebocyte Lysate (LAL) Kinetic-QCL Kit (Lonza, Basel, Switzerland) according to the

455 manufacturer's instructions. Purified phage preparations containing endotoxin units

456 (EU)/1E09 PFU $\leq 2$ were used for the phage therapy experiments. The phage stock was

457 stored at $4^{\circ} \mathrm{C}$ in the dark until use.

459 Acknowledgments:

We would like to thank Professor Mikael Skurnik for kindly providing the $\phi A 1122$ phage.

\section{References}

463 1. Inglesby, T. V.; Dennis, D.T.; Henderson, D.A.; Bartlett, J.G.; Ascher, M.S.; Eitzen,

464 E.; Fine, A.D.; Friedlander, A.M.; Hauer, J.; Koerner, J.F.; et al. Plague as a 
467 2. Pechous, R.D.; Sivaraman, V.; Stasulli, N.M.; Goldman, W.E. Pneumonic Plague:

3. Sebbane, F.; Lemaître, N. Antibiotic therapy of plague: A review. Biomolecules

471 2021, 11.

4. Rotem, S.; Steinberger-Levy, I.; Israeli, O.; Zahavy, E.; Aloni-Grinstein, R. Beating the bio-terror threat with rapid antimicrobial susceptibility testing.

5. Randremanana, R.; Andrianaivoarimanana, V.; Nikolay, B.; Ramasindrazana, B.; Paireau, J.; ten Bosch, Q.A.; Rakotondramanga, J.M.; Rahajandraibe, S.; urban plague epidemic in Madagascar, August-November, 2017: an outbreak report. Lancet Infect. Dis. 2019, 19, 537-545, doi:10.1016/S1473-3099(18)30730-

6. Rabaan, A.A.; Al-Ahmed, S.H.; Alsuliman, S.A.; Aldrazi, F.A.; Alfouzan, W.A.; breaks usual seasonal mould. J. Med. Microbiol. 2019, 68, 292-302, doi:10.1099/jmm.0.000915. 
487 8. Guiyoule, A.; Gerbaud, G.; Buchrieser, C.; Galimand, M.; Rahalison, L.; Chanteau,

9. Galimand, M.; Carniel, E.; Courvalin, P. Resistance of Yersinia pestis to antimicrobial agents. Antimicrob. Agents Chemother. 2006, 50, 3233-3236, doi:10.1128/AAC.00306-06.

10. Welch, T.J.; Fricke, W.F.; McDermott, P.F.; White, D.G.; Rosso, M.L.; Rasko, D.A.;

11. Andrianaivoarimanana, V.; Wagner, D.M.; Birdsell, D.N.; Nikolay, B.;

Rakotoarimanana, F.; Randriantseheno, L.N.; Vogler, A.J.; Sahl, J.W.; Hall, C.M.;

12. Moore, B.D.; Macleod, C.; Henning, L.; Krile, R.; Chou, Y.L.; Laws, T.R.; Butcher, vaccination in a pneumonic plague model. Vaccines 2022. modern biology. BioEssays 2015, 37, 6-9, doi:10.1002/bies.201400152. 
508 14. d'Herelle, F. Bacteriophage as a Treatment in Acute Medical and Surgical Infections. Bull. N. Y. Acad. Med. 1931, 7, 329-48.

15. Kortright, K.E.; Chan, B.K.; Koff, J.L.; Turner, P.E. Phage Therapy: A Renewed

16. Liu, D.; Van Belleghem, J.D.; de Vries, C.R.; Burgener, E.; Chen, Q.; Manasherob, 219-232, doi:10.1016/j.chom.2019.01.014.

17. Nir-Paz, R.; Gelman, D.; Khouri, A.; Sisson, B.M.; Fackler, J.; Alkalay-Oren, S.;

18. Schooley, R.T.; Biswas, B.; Gill, J.J.; Hernandez-Morales, A.; Lancaster, J.; Lessor,

Khalifa, L.; Rimon, A.; Yerushalmy, O.; Bader, R.; et al. Successful Treatment of Antibiotic-resistant, Poly-microbial Bone Infection With Bacteriophages and Antibiotics Combination. Clin Infect Dis 2019, 69, 2015-2018, doi:10.1093/cid/ciz222. 
1809.

530

531

532

533

20. Gur, D.; Glinert, I.; Aftalion, M.; Vagima, Y.; Levy, Y.; Rotem, S.; Zauberman, A.; Tidhar, A.; Tal, A.; Maoz, S.; et al. Inhalational gentamicin treatment is effective against Pneumonic Plague In A Mouse Model. Front. Microbiol. 2018, 9, 1-10,

21. Byrne, W.R.; Welkos, S.L.; Pitt, M.L.; Davis, K.J.; Brueckner, R.P.; Ezzell, J.W.; doi:10.3389/fmicb.2018.00741.

22. Zauberman, A.; Gur, D.; Levy, Y.; Aftalion, M.; Vagima, Y.; Tidhar, A.; Chitlaru, T.; Mamroud, E. Postexposure administration of a Yersinia pestis live vaccine for potentiation of second-line antibiotic treatment against pneumonic plague. J. Infect. Dis. 2019, 220, 1147-1151.

23. d'Herelle, F. Essai de traitement de la peste bubonique par le bacteriophage. Press. Med 1925, 33, 1393-1394.

24. Filippov, A.A.; Sergueev, K. V.; He, Y.; Huang, X.Z.; Gnade, B.T.; Mueller, A.J.; Fernandez-Prada, C.M.; Nikolich, M.P. Bacteriophage therapy of experimental bubonic plague in mice. In Proceedings of the Advances in Experimental Medicine and Biology; de Almeida, A.M.P., Leal, N.C., Eds.; Springer New York: New York, NY, 2012; Vol. 954, pp. 337-348. 
549 25. Filippov, A.A.; Sergueev, K. V; He, Y.; Huang, X.-Z.; Gnade, B.T.; Mueller, A.J.; 6, e25486.

26. Zhao, X.; Skurnik, M. Bacteriophages of Yersinia pestis. In Yersinia pestis:

2016; Vol. Advances i, pp. 361-375 ISBN 9789402408904.

27. Chu, C.M. Laboratory Manual of Plague Diagnostic Tests; Center for Disease

28. Moses, S.; Vagima, Y.; Tidhar, A.; Aftalion, M.; Mamroud, E.; Rotem, S.;

29. Frati, K.; Malagon, F.; Henry, M.; Delgado, E.V.; Hamilton, T.; Stockelman, M.G.;

human whole blood for the selection of efficient therapeutic phages. Viruses

30. Ma, L.; Green, S.I.; Trautner, B.W.; Ramig, R.F.; Maresso, A.W. Metals Enhance the doi:10.1038/s41598-018-20698-2.

31. Dąbrowska, K.; Abedon, S.T. Pharmacologically aware phage therapy: 
pharmacodynamic and pharmacokinetic obstacles to phage antibacterial action in

32. Filippov, A.A.; Sergueev, K. V; Nikolich, M.P. Can phage effectively treat doi:10.4161/bact.22407.

33. Tagliaferri, T.L.; Jansen, M.; Horz, H.-P. Fighting Pathogenic Bacteria on Two Fronts: Phages and Antibiotics as Combined Strategy. Front. Cell. Infect. Microbiol.

34. Li, X.; He, Y.; Wang, Z.; Wei, J.; Hu, T.; Si, J.; Tao, G.; Zhang, L.; Xie, L.; Abdalla, A.E. 2019, 9, doi:10.3389/fcimb.2019.00022.

35. Menor-Flores, M.; Vega-Rodríguez, M.A.; Molina, F. Computational design of

36. Heine, H.S.; Hershfield, J.; Marchand, C.; Miller, L.; Halasohoris, S.; Purcell, B.K.;

Worsham, P.L. In vitro antibiotic susceptibilities of Yersinia pestis determined by

59, 1919-1921.

37. Steinberger-Levy, I.; Shifman, O.; Zvi, A.; Ariel, N.; Beth-Din, A.; Israeli, O.; Gur, D.; 
pestis susceptibility to ciprofloxacin by the quantification of differentially doi:10.3389/fmicb.2016.00763.

38. Kiljunen, S.; Datta, N.; Dentovskaya, S. V; Anisimov, A.P.; Knirel, Y.A.; Bengoechea, J.A.; Holst, O.; Skurnik, M. Identification of the lipopolysaccharide core of Yersinia pestis and Yersinia pseudotuberculosis as the receptor for bacteriophage $\phi A 1122$.

39. Vagima, Y.; Zauberman, A.; Levy, Y.; Gur, D.; Tidhar, A.; Aftalion, M.; Shafferman, e1004893, doi:10.1371/journal.ppat.1004893.

40. Levy, Y.; Vagima, Y.; Tidhar, A.; Aftalion, M.; Gur, D.; Nili, U.; Chitlaru, T.; 

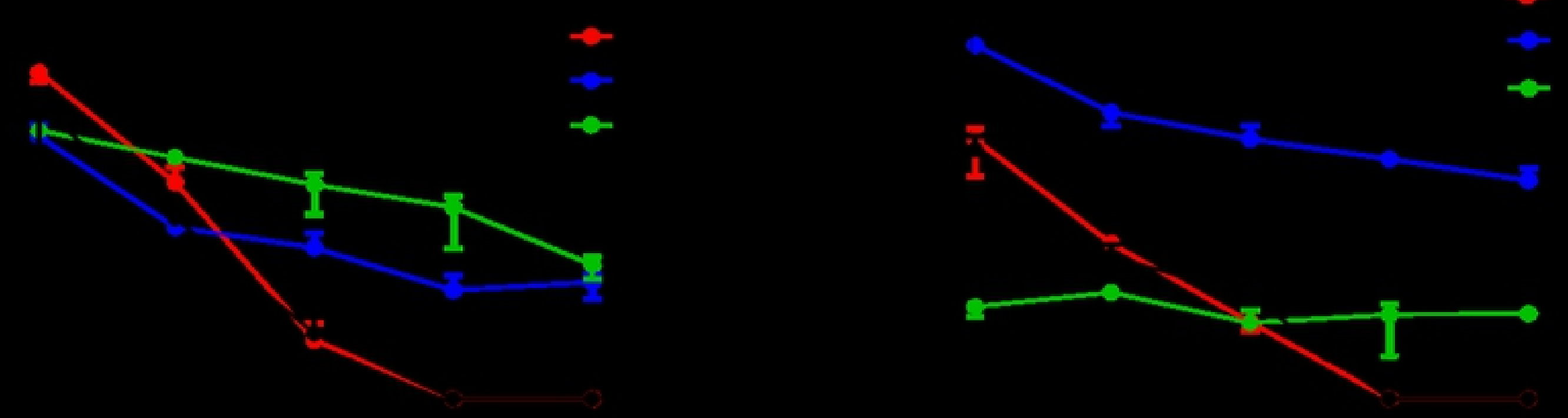

Figure 1 


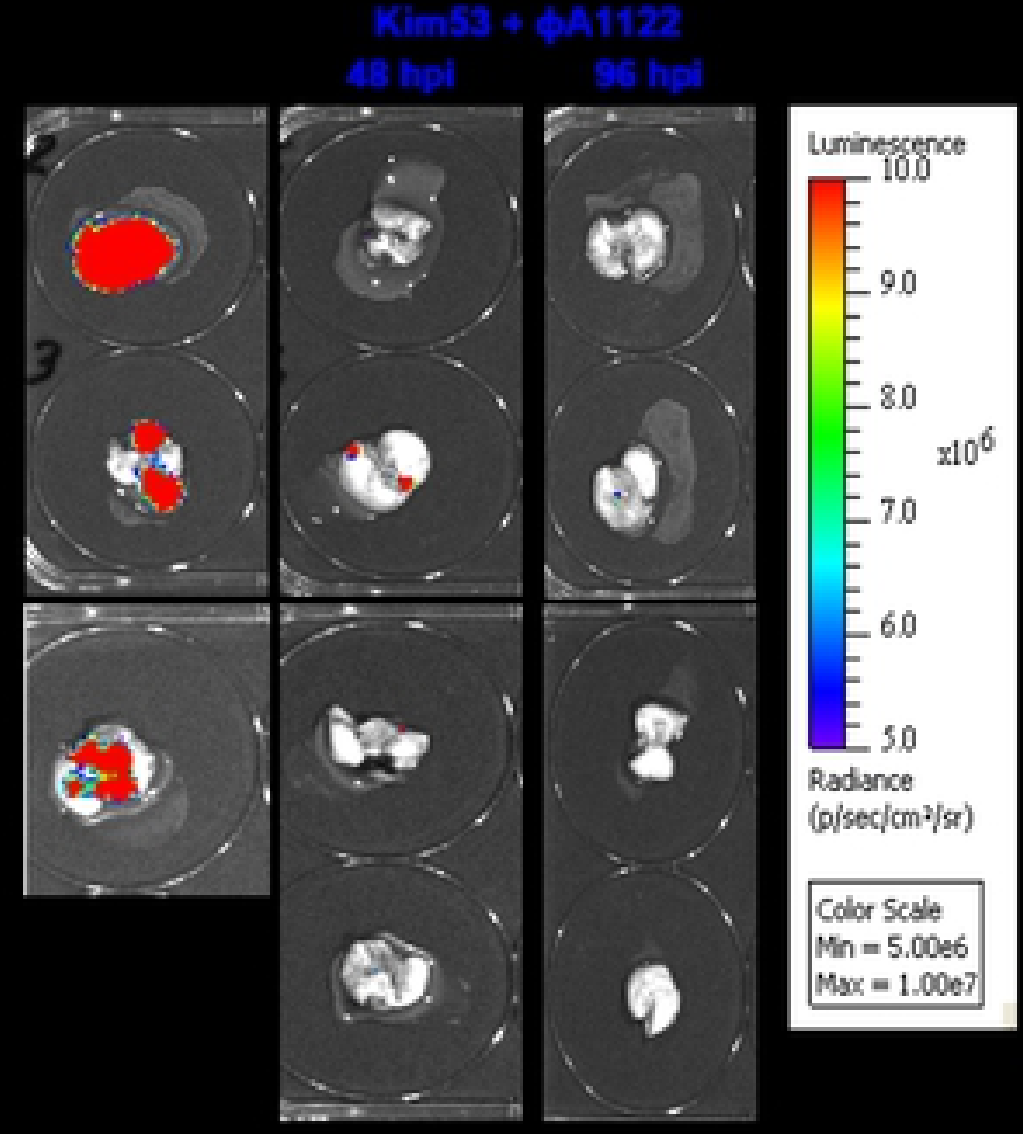

Figure 2 
230

23.

23 g.

23 igs:

23 gas:
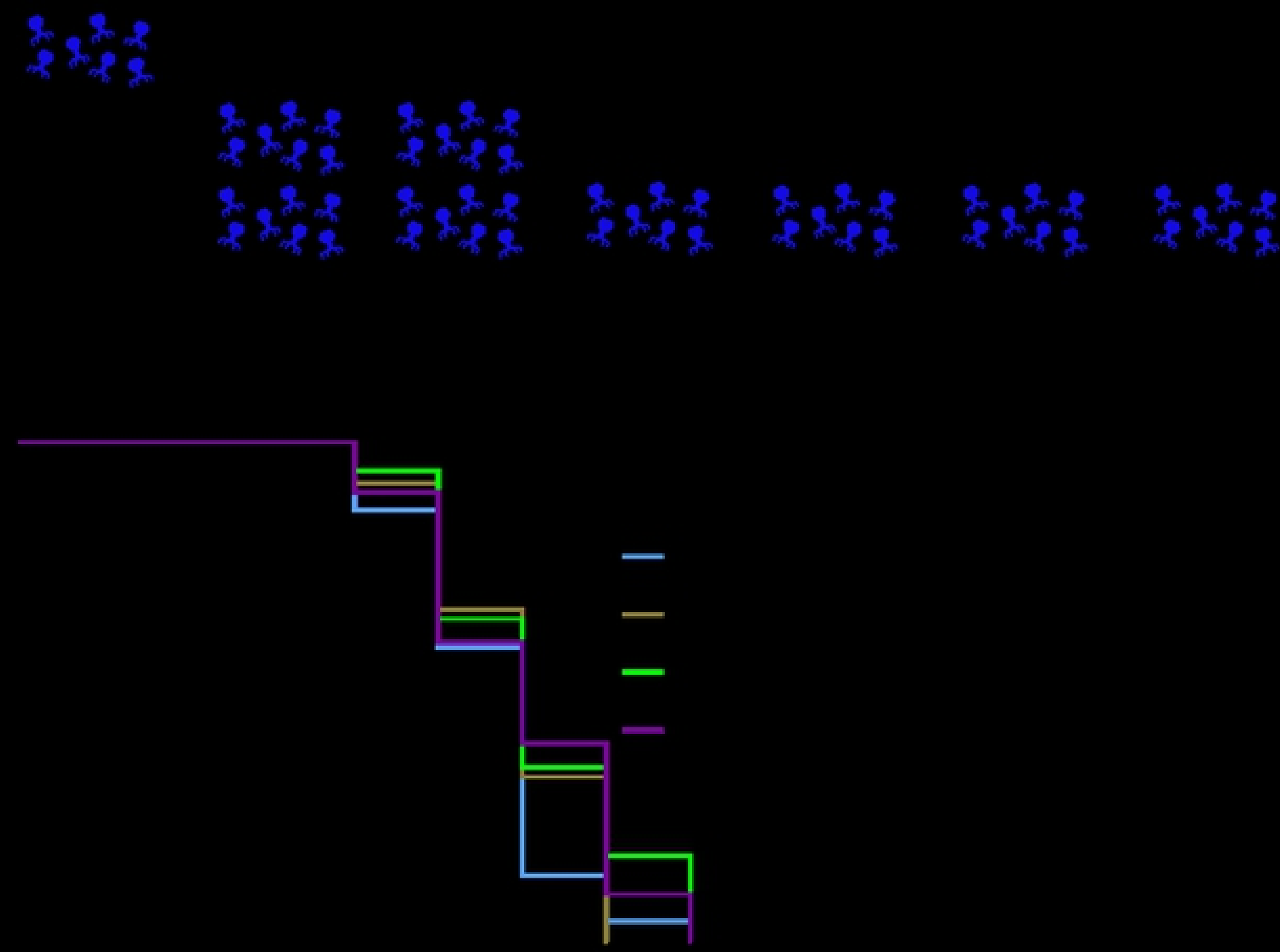

Figure 3 


\section{\$A1122 I}

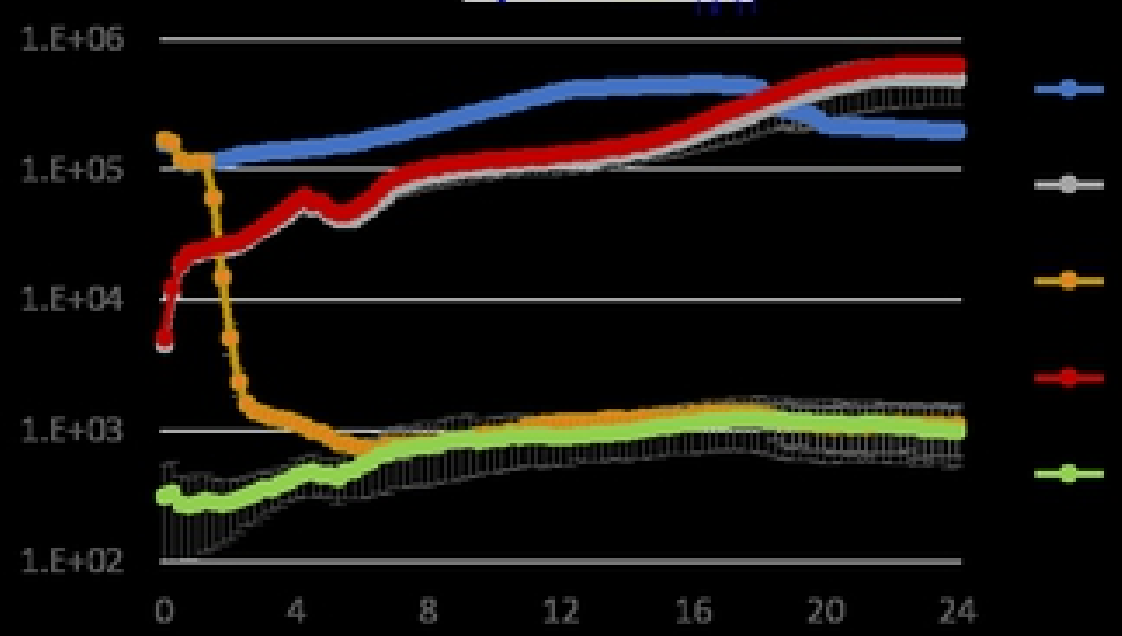

PST P

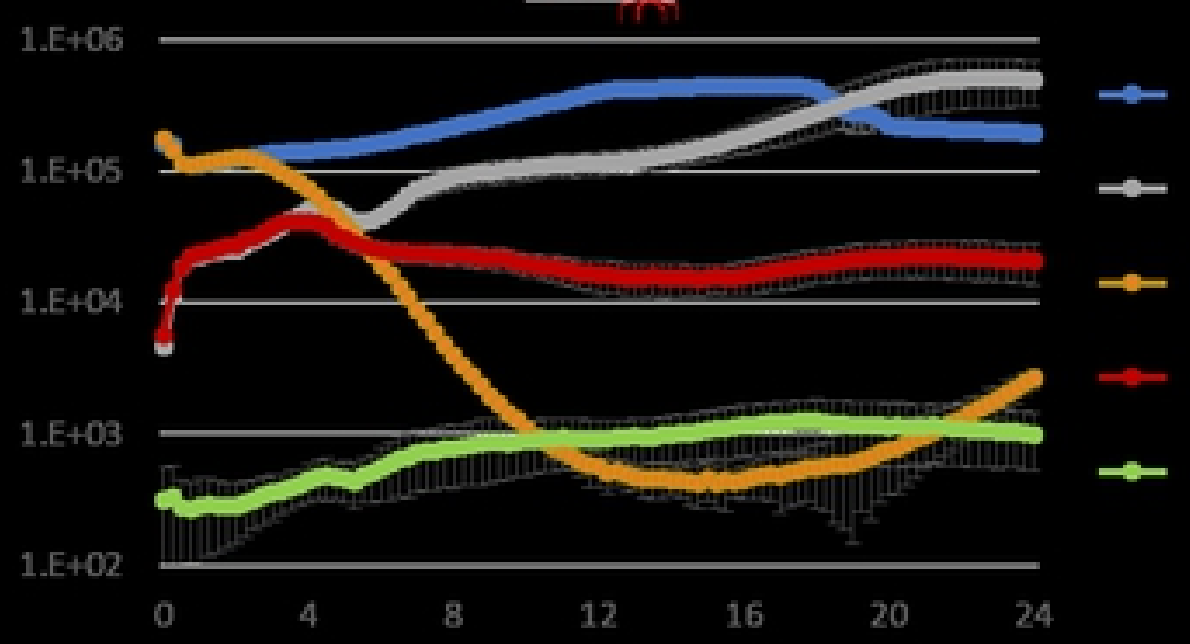

Figure 4 

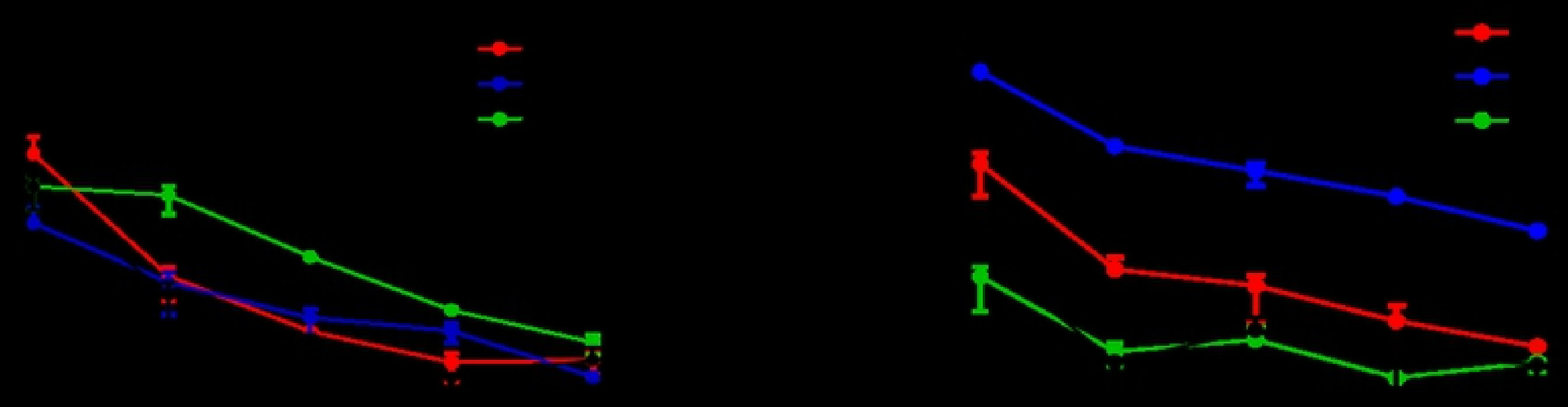

Figure 5 
a

Cusin

23.
के

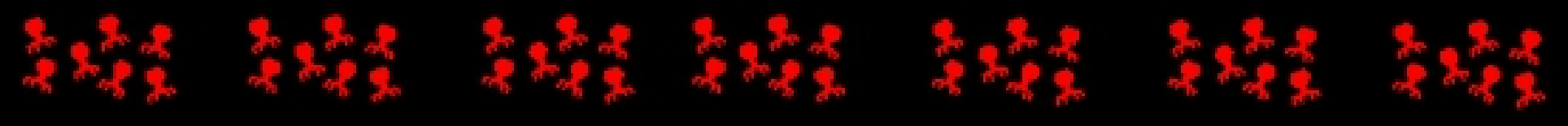

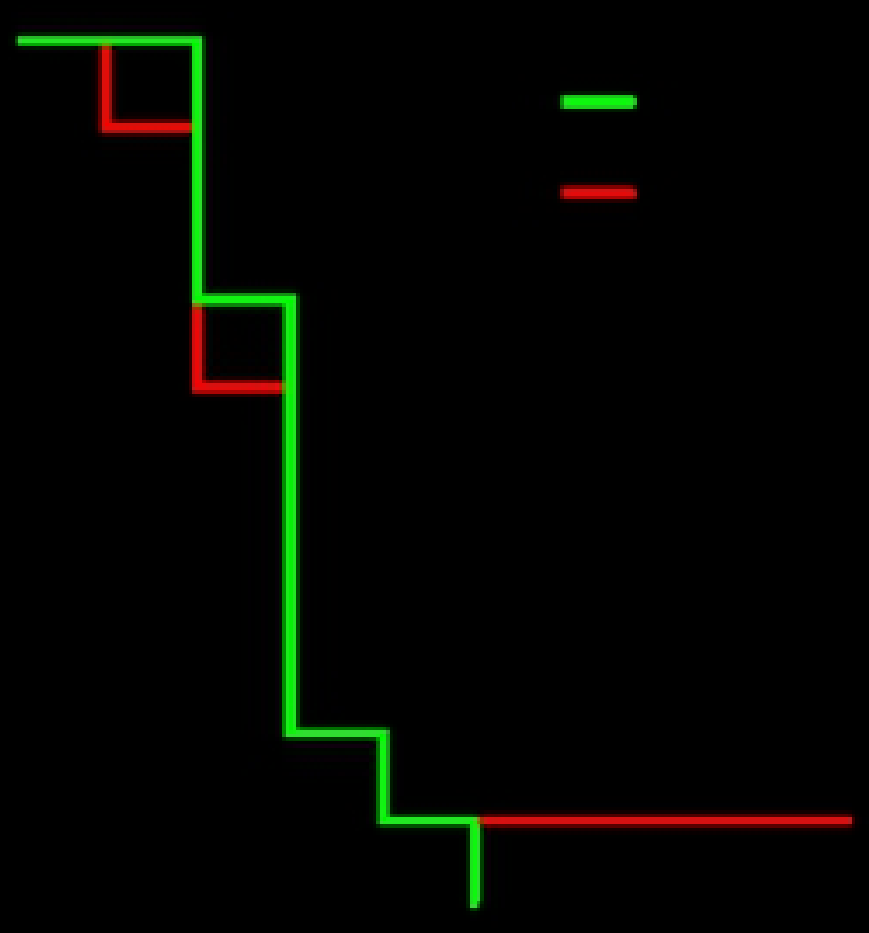

Figure 6 
